\title{
Philosophiques
}

\section{Les troubles psychiatriques et le modèle des espèces pratiques}

\section{Peter Zachar}

Volume 33, numéro 1, printemps 2006

Philosophie et psychopathologie

URI : https://id.erudit.org/iderudit/012948ar

DOI : https://doi.org/10.7202/012948ar

Aller au sommaire du numéro

\section{Éditeur(s)}

Société de philosophie du Québec

\section{ISSN}

0316-2923 (imprimé)

1492-1391 (numérique)

Découvrir la revue

\section{Citer cet article}

Zachar, P. (2006). Les troubles psychiatriques et le modèle des espèces pratiques. Philosophiques, 33(1), 81-97. https://doi.org/10.7202/012948ar

\section{Résumé de l'article}

Cet article explore la classification des troubles psychiatriques dans la perspective du modèle des espèces pratiques. En nous basant sur certains travaux en philosophie des sciences qui soutiennent que les éléments chimiques et les espèces biologiques ne possèdent pas de véritables essences, nous affirmons que les troubles psychiatriques ne devraient pas être compris, eux non plus, de façon essentialiste. Les troubles psychiatriques sont des " espèces pratiques ", non des " espèces naturelles ". Ce modèle représente une approche pragmatiste de la classification qui pose, compte tenu de l'infinie complexité du monde, qu'une multitude de décisions peuvent jouer un rôle légitime dans la définition des limites des catégories. Il s'agit d'une approche nominaliste contemporaine qui considère les concepts et les catégories comme provisoires et partiels, sans toutefois rejeter l'idée qu'ils sont des espèces légitimes. Ce modèle rejette ce que Ian Hacking a nommé le structurisme inhérent, sans pour autant rejeter les espèces. Le modèle des espèces pratiques rejette aussi l'éliminativisme. Il est en accord avec les arguments non essentialistes offerts par les éliminativistes, mais il n'accepte pas leurs conclusions austères. Je discute finalement les complications et les imperfections du modèle. 


\title{
Les troubles psychiatriques et le modèle des espèces pratiques
}

\author{
PETER ZACHAR \\ Auburn University Montgomery \\ pzachar@mail.aum.edu
}

\begin{abstract}
RÉSUMÉ. - Cet article explore la classification des troubles psychiatriques dans la perspective du modèle des espèces pratiques. En nous basant sur certains travaux en philosophie des sciences qui soutiennent que les éléments chimiques et les espèces biologiques ne possèdent pas de véritables essences, nous affirmons que les troubles psychiatriques ne devraient pas être compris, eux non plus, de façon essentialiste. Les troubles psychiatriques sont des « espèces pratiques », non des « espèces naturelles ». Ce modèle représente une approche pragmatiste de la classification qui pose, compte tenu de l'infinie complexité du monde, qu'une multitude de décisions peuvent jouer un rôle légitime dans la définition des limites des catégories. Il s'agit d'une approche nominaliste contemporaine qui considère les concepts et les catégories comme provisoires et partiels, sans toutefois rejeter l'idée qu'ils sont des espèces légitimes. Ce modèle rejette ce que lan Hacking a nommé le structurisme inhérent, sans pour autant rejeter les espèces. Le modèle des espèces pratiques rejette aussi l'éliminativisme. Il est en accord avec les arguments non essentialistes offerts par les éliminativistes, mais il n'accepte pas leurs conclusions austères. Je discute finalement les complications et les imperfections du modèle.
\end{abstract}

\begin{abstract}
This article explores the classification of psychiatric disorders from the perspective of the practical kinds model. Based on work in the philosophy of science which argues that chemical elements and biological species do not have real essences, it is argued that psychiatric disorders should not be understood essentialistically either. Psychiatric disorders are practical, not natural kinds. This model represents a pragmatist approach to classification which holds that, because the world is so complex, a multitude of decisions can play legitimate roles in defining category boundaries. It is a contemporary nominalistic approach that views concepts and categories as provisional and partial, but does not reject the notion that there are legitimate kinds. It rejects what lan Hacking has called inherent structurism, but not kinds. The practical kinds model is also anti-eliminativist. It agrees with the non-essentialistic arguments offered by eliminativists, but does not accept their austere conclusions. Complications and shortcomings of the model are discussed.
\end{abstract}

\section{Introduction}

Le modèle des espèces pratiques adopte une vue pluraliste des catégorisations scientifiques et garde l'esprit ouvert quant à la validité de tout schéma de classification particulier. Classifier implique de faire des distinctions. On fait des distinctions parce que celles-ci sont censées être utiles, mais ce qui constitue une distinction utile dans un contexte l'est moins dans un autre. Les distinctions doivent donc être évaluées en fonction de leur capacité à résoudre des problèmes. 
Dans cet article, je vais passer en revue les principes fondamentaux du modèle des espèces pratiques tel qu'appliqué aux troubles psychiatriques, spécifiquement, les principes pertinents à la philosophie des sciences et à la philosophie de la psychiatrie. Ces principes sont le non-essentialisme, le respect de la complexité, le nominalisme, et un certain scepticisme concernant les mérites de l'éliminativisme.

\section{Une approche non essentialiste des troubles psychiatriques}

Le modèle des espèces pratiques peut être compris en le comparant aux autres modèles de la maladie mentale, particulièrement au modèle des problèmes de vie [problems-in-living model], au modèle du matérialisme biomédical du "cerveau brisé " [broken brain] et au modèle du dysfonctionnement préjudiciable. Les partisans de ces modèles pensent tous qu'une véritable maladie mentale est une espèce naturelle. Les exemples d'espèces naturelles comprennent traditionnellement des entités comme les maladies et les espèces biologiques - dont on considère qu'elles possèdent une essence.

Selon le modèle des problèmes de vie, ce que l'on nomme maladie mentale représente des difficultés à faire face et à s'ajuster aux demandes de la vie. C'est le modèle qui a la cote parmi les psychologues humanistes comme Laing (1960). C'est aussi celui que préfère Thomas Szasz (1961) et les antipsychiatres, qui déclarent que la schizophrénie n'est pas une maladie comme l'est la tuberculose. Ils croient que la tuberculose peut être identifiée à un pathogène particulier et constitue donc une espèce naturelle mais que la schizophrénie, qui ne peut pas être identifiée par un pathogène spécifique, n'est pas une espèce naturelle et n'est donc pas une maladie mentale légitime. Selon les anti-psychiatres, la schizophrénie représente un type de déviance sociale défini par un groupe de symptômes qui interfère avec la vie quotidienne de chacun, plutôt qu'une pathologie.

Le modèle du cerveau brisé pose que les maladies mentales sont des troubles cérébraux, et comme telles, sont des espèces naturelles (Andreasen, 1984 ; Guze, 1992). D'après ce modèle, l'essence de toute maladie mentale est un processus biologique pathologique. Les tenants de ce modèle croient qu'il est important de reconnaître la nature physique de la maladie mentale pour établir et améliorer les traitements médicaux. En réponse aux antipsychiatres et aux penseurs sympathisant avec l'humanisme, les partisans du modèle du matérialisme biomédical maintiennent que les activités pathologiques sont des choses complexes. La tuberculose est un exemple de maladie infectieuse, mais il existe d'autres espèces de maladies. Les partisans de ce modèle déclarent aussi que les affirmations des anti-psychiatres sur l'incapacité des médecins à guérir quelque maladie mentale que ce soit ne sont pas pertinentes pour son statut d'entité pathologique. Il est fondamentalement établi que la médecine peut contrôler certains troubles médicaux légitimes, mais non pas les guérir. Ces troubles sont le diabète, l'hypertension artérielle essentielle, les troubles bipolaires, la dépression et la schizophrénie. 
Le modèle du dysfonctionnement préjudiciable de Jerome Wakefield $(1992,2000)$ est censé aider les psychiatres et les psychologues à distinguer entre les conditions telles qu'un comportement mésadapté, comme le deuil chronique, de celles qui sont de "véritables " troubles, comme la dépression mélancolique. Ce dernier croit que ce modèle sous-tend de fortes intuitions concernant ce qu'est un trouble psychiatrique légitime. L'idée principale derrière le modèle de Wakefield est qu'une maladie mentale a deux composantes. La première est son caractère préjudiciable - qui est similaire à ce que les antipsychiatres ont appelé les problèmes de vie. Le comportement en question est évalué comme étant une inadaptation de l'individu. La seconde est la dysfonction, c'est-à-dire une défectuosité de la machinerie cognitive-émotionnelle-perceptuelle de l'organisme. Elle ne fonctionne pas de la façon pour laquelle a été conçue. Les fonctions naturelles sont supposées posséder des "essences cachées » (black block essences), ce qui signifie que les essences structurelles de Putnam et Kripke sont présentes même si nous ne les avons pas identifiées.

Le modèle des espèces pratiques a beaucoup en commun avec chacun de ces modèles, mais contrairement à eux, il refuse d'affirmer que les troubles psychiatriques légitimes doivent avoir de véritables essences (Zachar, 2000a, $2000 \mathrm{~b}$ ). Selon le modèle des espèces pratiques, les troubles psychiatriques répertoriés dans le Diagnostic and Statistical Manual of Mental Disorders (DSM) et le International Classification of Diseases (ICD) sont des états cognitifs, affectifs, biologiques et comportementaux qui sont véritables et distincts. Il existe, certes, des raisons pour lesquelles les psychiatres les ont inclus dans les manuels diagnostiques, mais ce que tous ces états ont en commun, en tant que troubles psychiatriques, c'est d'être dans ces manuels. Plusieurs regroupements de ces troubles ont bien d'autres choses en commun, mais le fait d'être dans le manuel constitue le lien principal entre ces états.

Il est important de souligner que l'inclusion dans le manuel ne constitue pas une justification pour considérer qu'une condition spécifique est un trouble psychiatrique - et les troubles qui sont classés dans le manuel doivent y être pour des raisons légitimes. Il y a certains troubles qui sont paradigmatiques, comme la schizophrénie et la dépression clinique, d'autres dont l'inclusion dans les manuels provoque des débats, tels que les troubles d'adaptation et les troubles oppositionnels avec provocation, et des conditions qui sont au-delà des frontières floues de la catégorie des troubles psychiatriques, comme celles qui figurent sous le code $\mathrm{V}$, par exemple les problèmes liés à l'acculturation.

Puisque le modèle des espèces pratiques reconnaît qu'identifier une prédisposition génétique ou un marqueur biologique peut jouer un rôle légitime dans les décisions concernant l'organisation du manuel, il n'est pas une version du constructionnisme social. Il n'est pas non plus une version du modèle du cerveau brisé, car il permet que les types d'analyse psychologique et relationnelle puissent aussi nous aider à décider de l'organisation du manuel, et, dans certains cas, à négliger les analyses de type biologique. Ses 
partisans sont sceptiques quant aux tentatives d'organiser le manuel en utilisant un seul type d'analyse, et ils rejettent fermement toute proposition philosophique qui accorde une priorité ontologique à un type d'analyse particulier. Finalement, le modèle du dysfonctionnement préjudiciable est utile pour distinguer un trouble de ce qui n'en est pas, mais une solution au problème de la discrimination n'est pas la seule considération dont nous devons tenir compte lorsque nous décidons de ce qui est le contenu approprié des manuels diagnostiques.

Le modèle des espèces pratiques cherche à rendre possible que les troubles de croyances, des désirs, des émotions, du soi et de la personnalité soient des espèces scientifiquement respectables. Ces troubles ne sont pas des espèces naturelles, mais ils ne devraient pas être dévalués et considérés comme arbitraires ou artificiels. Les espèces pratiques sont plus que des expédients que l'on utilise lorsque nous ne savons pas quoi faire d'autre et ne sont pas non plus des indications nous menant vers les espèces naturelles. Si nous acceptons que les diagnostics sont des outils qui nous aident à interagir avec le monde " réel ", alors ils doivent être justifiés par la façon dont ils parviennent à remplir leur fonction. C'est tout ce qu'il est nécessaire d'établir, et c'est cela, être une espèce pratique.

\section{Pourquoi le non-essentialisme ?}

Les véritables essences font d'une chose ce qu'elle est, et les propriétés importantes de cette chose sont censées découler de cette essence. Les essences sont habituellement conçues comme les propriétés microstructurelles intrinsèques telles que le nombre de protons dans le noyau des éléments chimiques et l'ADN d'une espèce biologique.

Le problème des véritables essences, c'est qu'elles n'ont pas été découvertes par la science et ne pourraient l'être. Les scientifiques ont découvert des propriétés nécessaires, mais bien qu'elles soient essentielles, elles ne sont pas des essences. Par exemple, les lobes frontaux sont nécessaires à la rationalité, et un certain type d'activité amygdalienne est nécessaire à l'émotion de la peur, mais ce ne sont pas des essences. Une essence est censée être la propriété causale la plus importante, et celle qui est variétale [sortal]. Au regard de la causalité, de nombreuses propriétés différentes sont importantes pour la production de phénomènes complexes, mais, pour des raisons de simplicité, elles sont souvent réduites à des clauses ceteris paribus. Pour ce qui est de l'identification, les propriétés intrinsèques ne déterminent pas à elles seules les limites de la catégorie. Même si les propriétés intrinsèques sont de toute évidence importantes, les critères externes peuvent toujours influer sur ce que nous croyons être les catégories pertinentes.

Il existe plusieurs bons arguments philosophiques qui montrent que les éléments chimiques ou les espèces n'ont pas de véritables essences. Selon le modèle des espèces pratiques, si les éléments et les espèces n'ont pas de véritables essences, les troubles psychiatriques n'ont pas à en avoir non plus. 
En ce qui concerne les éléments chimiques, Bartlett et moi-même (2001) avons souligné que les scientifiques ont découvert que le noyau de l'hydrogène contient un proton mais ont décidé que posséder un noyau est l'essence de l'hydrogène. Des arguments similaires ont été donnés par Canfield (1983), Donnellan (1983), and Bryant (2000). Bien que, par convention, nous classions l'hydrogène et ses divers isotopes comme étant le même élément, nous pourrions logiquement les classer comme des éléments reliés, mais différents. Par exemple, si c'était le nombre d'isotopes qui constituait la convention acceptée pour définir les éléments chimiques, certaines choses considérées maintenant comme de l'hydrogène ne le seraient plus. Des différences fortes et importantes au point de vue pratique entre un élément et ses isotopes fourniraient des raisons pour considérer ceux-ci comme des espèces différentes.

C'est clairement le cas avec l'hydrogène. Il existe des différences pratiques importantes entre l' « hydrogène » et ses isotopes, comme le deutérium et le tritium, un cas où posséder un seul proton est secondaire par rapport au comportement des isotopes. Pour les chimistes professionnels, le deutérium est un lexème premier autant qu'un terme variétal. Il est vrai que pour la plupart des fins pratiques, il n'y a pas de différences importantes entre un "élément conventionnel » et ses isotopes; parfois, cependant, les différences de la masse et de la durée de la décroissance radioactive peuvent devenir importantes dans la pratique. Cela signifie que non seulement la structure inhérente mais les facteurs externes ou « relationnels » sont importants dans la décision concernant ce que nous considérerons être une espèce. Le nombre de protons dans le noyau ne définit pas, en soi, ce qu'est une espèce ni ne détermine la " nature " de celle-ci - ce n'est pas une essence.

Quant aux espèces animales, nous avons souligné, avec Ghaemi (2000a, sous presse), que la possibilité même de l'évolution fait que les propriétés d'une espèce peuvent changer graduellement et que ce qui est une propriété mineure à un moment donné peut en venir à dominer un groupe ultérieurement. Cela est également vrai en ce qui concerne l'ADN. Contrairement à Linné, avec son modèle essentialiste dans lequel les membres d'une espèce partagent une essence commune, Darwin a défini une espèce comme une population d'individus uniques. Une population est un système génétique, comportemental et écologique dont les membres luttent entre eux, et, lorsque réunis en un tout, contre les membres d'autres espèces. Ainsi, la variation individuelle, plutôt qu'être ce qu'un essentialiste nommerait une "imperfection ", une " erreur " ou un " accident ", devient indispensable à la compréhension à long terme de la nature fluide des espèces. Il existe donc une relative stabilité, mais pas d'essence immuable.

Un autre argument en faveur du non-essentialisme est davantage d'ordre psychologique. Des recherches en psychologie du développement démontrent que les enfants, dès l'âge de quatre ans, sont naturellement enclins à voir les espèces biologiques de façon essentialiste, c'est-à-dire qu'ils pensent posséder des propriétés intrinsèques qui font d'eux ce qu'ils sont (Gelman et Coley, 
1990 ; Gelman, et Wellman, 1991, Gelman et Hirschfeld, 1999). Ce biais essentialiste est présent et perdure dans la pensée adulte. L'essentialisme s'avère donc être le point de vue du sens commun au cœur de la biologie populaire, sans être pour autant limité à celle-ci. Bien qu'il soit utile dans le quotidien, il n'est pas nécessairement utile sur le plan scientifique, et concevoir des catégories de façon non essentialiste peut être contre-intuitif. Apprendre à devenir un scientifique exige souvent l'intégration de propositions contre-intuitives dans le modèle de travail du domaine en question - et cela vaut pour la psychiatrie et la psychologie comme pour la chimie et la biologie.

\section{Les espèces pratiques et la complexité}

Le monde est complexe, et de nombreux facteurs entrent dans la décision du type de catégorie à adopter pour le décrire. Pour le décrire nous pouvons mettre les choses ensemble et les considérer comme la même espèce, ou les séparer et les voir comme des espèces différentes; la façon de procéder change selon les moments en fonction des problèmes que l'on tente de résoudre. Comme le suggérait Nelson Goodman (1978), les problèmes que nous devons résoudre affectent la manière dont nous rassemblons ou séparons les catégories. Ils affectent aussi la façon dont nous évaluons l'importance des propriétés, dont nous les ordonnons l'une par rapport à l'autre, dont nous les simplifions en vue d'un usage plus aisé, et la façon dont nous décidons des propriétés qui seront ignorées ou déformées.

Un exemple de regroupement en psychiatrie peut être illustré à l'aide des différents concepts de troubles spectraux [spectrum disorder]. Le plus connu parmi les concepts de trouble spectral est la théorie selon laquelle les schizophrénies, les personnalités schizotypales, les personnalités paranoïaques et les troubles schizoaffectifs sont tous membres du "spectre schizophrénique ", c'est-à-dire membres d'une espèce cohérente au point de vue génétique. De la même façon, Wender et Klein (1982) suggèrent que l'alcoolisme, le comportement antisocial, les troubles de somatisation et l'hyperactivité infantile peuvent tous être attribués aux mêmes défauts dans le lobe frontal et le système limbique, plus spécifiquement à une incapacité à inhiber les tendances dominantes de réponse. Ils les ont nommés " le quartette sans nom ».

Un exemple de catégorie qui a été fragmentée peut être illustré par un travail récent sur l'autisme. Pour des raisons liées au pronostic, les auteurs du DSM-IV ont décidé de diviser l'autisme en catégories séparées. La première distingue l'autisme proprement dit d'une forme moins sévère d'autisme connue sous le nom de désordre d'Asperger. Le trouble d'Asperger peut aussi être moins stigmatisant, un facteur important en sa faveur. Basé sur la décision des Européens quant au développement du ICD, les auteurs du DSMIV ont séparé les formes plus pathologiques des troubles envahissants du développement en autisme, le syndrome de Rett et le trouble désintégratif de l'enfance. Les deux dernières conditions sont considérées comme des phénocopies sévères de l'autisme. Il existe des raisons pour séparer ce qu'il était 
convenu d'appeler autisme de cette façon, et on peut argumenter que chaque condition est une condition distincte correspondant à un type d'analyse biologique, psychologique ou relationnelle.

Plutôt que de rechercher une essence microstructurelle unique déterminant ce qui fera partie d'une catégorie, le modèle des espèces pratiques met en évidence une multitude de facteurs pertinents au choix des catégories. J'ai suggéré (2002) que les considérations pertinentes pour le choix des catégories comprennent : les traitements disponibles ; les stratégies potentielles de gestion; les effets de l'étiquetage ; la maximisation des vrais positifs et des vrais négatifs dans l'identification ; l'homogénéité à l'intérieur d'une même catégorie pour créer des groupes dans la recherche expérimentale ; le dévoilement de scénarios étiologiques (spécialement pour les troubles spectraux); le déroulement temporel du trouble ; la prédiction des diagnostics ; la démarcation entre trouble et pseudo-trouble ; la cohérence avec les sciences fondamentales en génétique, en physiologie et en psychologie; le fait d'être cliniquement informatif et d'une utilisation simple, et finalement de rencontrer les standards psychométriques comme la fiabilité et la validité. Sur un certain plan, ces choses sont toutes des pratiques. Nous faisons des choses avec les éléments d'une catégorie. Nous interagissons avec eux, et, sur la base de cette interaction, nous apprenons comment penser au sujet de la catégorie (ou à l'utiliser). Ces pratiques doivent également être aussi jaugées l'une par rapport à l'autre.

L'ouverture à la recatégorisation qui définit le modèle des espèces pratiques n'est pas conforme à l'approche classique des catégories. Dans l'approche classique, une catégorie est défendue en termes d'essence intrinsèque qui détermine sa nature et celle de ses éléments. Quelque chose est ou n'est pas un élément d'une catégorie. Les divers modèles de ressemblance de famille, comme les prototypes et les modèles exemplaires sont une solution de rechange à l'approche classique. Lakoff (1987) les nomme catégories radiales. Dans les catégories radiales, les degrés d'appartenance peuvent être échelonnés, et les frontières sont floues quant à ce qui appartient ou pas à une catégorie.

Un bon exemple de catégorie radiale est l'émotion de base ["basic emotion "], telle que la peur. L'émotion de base de la peur peut être définie par divers états exemplaires comme la peur des araignées, la peur du sang ou la peur de l'échec. Celles-ci ne sont pas la même espèce d'état puisque la pression sanguine chute si l'objet de la peur est le sang, mais pas si c'est une araignée ou un échec. Dans d'autres modèles, la panique est considérée comme l'état biologique exemplaire de la même manière que la rage est considérée comme un exemplaire de la colère ; la panique et la peur présentent aussi différents covariants faciaux, vocaux et posturaux. Plus une émotion se trouve éloignée des exemplaires, moins elle ressemble à l'émotion de base. Les émotions apparentées dans la famille de la peur comprennent des états similaires à la peur, comme l'inquiétude et le sursaut. Il y a aussi des états adjacents ou voisins comme l'appréhension, la suspicion et l'expérience de l'étrangeté. Un état voisin 
serait, p. ex., la surprise. En plus de désigner un état spécifique, la peur qualifie tout ce que les états de la famille de la peur ont en commun.

En ce qui concerne les troubles de la personnalité, Widiger et Francis (1994) notent que les critères sont tellement nombreux que des personnes peuvent être classées comme antisociales en ayant très peu de caractéristiques en commun. Ils soulignent qu'il existe 848 façons différentes de rencontrer les critères d'une personnalité antisociale et que la personnalité antisociale est une famille de troubles plutôt qu'un trouble spécifique. Widiger et Francis considèrent que c'est en cela que réside la faiblesse de l'approche catégorielle des troubles de la personne, mais c'est une faiblesse seulement pour ceux que les modèles des familles de ressemblance intéressent moins. La raison pour laquelle on peut envisager favorablement les modèles de familles de ressemblance, c'est qu'ils aident à codifier la complexité rencontrée par les praticiens lors des diagnostics.

Certains psychologues expérimentaux reconnaissent que les êtres humains pensent le monde en utilisant les modèles de familles de ressemblance, mais ils soulignent que penser ainsi ne signifie pas que le monde est réellement structuré de cette manière (Grove et Tellegen, 1991; Ortony et Turner, 1990). Ils ont raison. Les recherches qui démontrent que les gens conçoivent, de façon implicite, les catégories d'émotions de base ou les catégories de couleurs comme des catégories radiales n'impliquent pas que ceux-ci doivent les concevoir de cette manière.

Le modèle des espèces pratiques s'engage d'avantage et affirme, sur un plan philosophique, que les scientifiques devraient conceptualiser les maladies, les émotions et les troubles psychiatriques comme des catégories radiales, c'està-dire des catégories dont les degrés d'appartenance sont échelonnés, dont les exemplaires sont nombreux et distincts, et qui ont des limites floues. Évidemment, toutes les catégories ne sont pas structurées de façon similaire. Certaines possèdent plus ou moins d'exemplaires, différents niveaux d'échelonnage, et le caractère flou de leurs limites varie. En fin de compte, de toutes façons, la recherche scientifique n'a pas découvert d'essences réelles, et les expérimentations actuelles dans les laboratoires et dans le monde nous indiquent que souvent le monde est plus complexe que ce qui est décrit par nos modèles.

Tant que le modèle des espèces pratiques n'est pas utilisé de manière obscurantiste et qu'il tente d'augmenter la fiabilité et la validité du diagnostic où cela est possible et indiqué, il devrait permettre aux gens d'entrevoir de nouvelles possibilités et des solutions plutôt que d'être limité à une semi-réification des catégories telles qu'elles sont acceptées de façon conventionnelle à certains moments de l'histoire.

\section{Les espèces pratiques et le nominalisme}

Le modèle des espèces pratiques relève d'une approche nominaliste, mais il est important d'être clair à propos du type de nominalisme qu'il représente. Plutôt qu'à un nominalisme traditionnel, on a affaire à une version du nominalisme plus contemporain, et les deux ne devraient pas être confondus. 
Le nominalisme traditionnel est un "nom-isme ", ou un point de vue selon lequel il n'y a pas d'espèce réelle. Une espèce est un regroupement d'individus. Selon le nominalisme traditionnel, seuls les individus ou les choses particulières existent véritablement. Cela constitue d'abord un rejet de l'essentialisme platonicien et aristo-thomiste qui soutient que l'essence sous-jacente d'un groupe a plus d'importance ontologique ou plus de "réalité " que les individus, ces derniers étant temporaires et transitoires. Rejeter l'existence des essences sous-jacentes qui font d'une personne un membre d'un groupe tel que homo sapiens ou patients schizophrènes suggère que "êtres humains " et "schizophrènes " ne sont rien d'autre que des noms désignant un regroupement d'individus. Les individus sont réels, mais pas les espèces. Le nominalisme traditionnel donne la priorité à l'unicité plutôt qu'à la ressemblance [sameness].

Le nominalisme contemporain consiste en un rejet encore plus fort de l'essentialisme et refuse d'associer la réalité d'une espèce avec la présence ou l'absence d'une quelconque essence. Du point de vue de la position nominaliste contemporaine, les essences n'ont rien à voir avec la question de la réalité d'une espèce. Elles ont été, selon J. S. Mill (1843/1973), une erreur philosophique qui a été difficile à éliminer.

Une erreur fondamentale est rarement évincée de la philosophie à la suite d'une seule victoire. Elle se retire lentement, défend chaque pouce de terrain et souvent, après avoir été chassée des aires ouvertes, elle continue de garder prise dans quelque coin reculé (p. 114).

Deux intuitions sont communément invoquées par ceux qui favorisent le nominalisme contemporain. La première intuition est que le monde est plus complexe, diversifié et singulier que ce que capturent nos catégories. Les catégories que nous adoptons simplifient inévitablement les choses, aplanissent les incohérences et ignorent les anomalies. Si, comme le dit Adorno, l'objet élude invariablement le concept, alors les catégories adoptées sont toujours provisoires, et davantage de preuves peuvent mener à une recatégorisation importante. Plus cette intuition est forte, plus l'on est enclin au nominalisme. Pour les nominalistes contemporains, toutefois, s'occuper de l'unicité ne requiert pas d'ignorer la ressemblance. Quoique les nominalistes contemporains la respectent de plus en plus, la distinction ressemblance/similarité [sameness/ similarity] reste considérée comme une notion un peu confuse.

La seconde intuition partagée par les nominalistes est que les " données » qui peuvent mener à une recatégorisation doivent être évaluées au regard de la tradition intellectuelle, des objectifs de l'enquête et du but recherché. Par exemple, si l'objectif de la recherche est la fiabilité du diagnostic, une catégorie comme la schizophrénie sera définie de façon très étroite. Le désavantage d'adopter des modèles avec une bande passante étroite et de haute-fidélité, c'est qu'il y aura de nombreuses exceptions et anomalies - donc des cas qui ne s'accorderont pas avec le modèle. La tendance veut qu'il n'y ait pas de 
catégories pour ces exceptions, ou bien, plus souvent, que le nombre de catégories prolifère. La recherche scientifique, on le comprend, rend la psychiatrie minutieuse. Adopter des modèles avec une bande passante étroite est une conséquence naturelle de la spécialisation. En même temps, il n'est pas pratique de laisser ce processus continuer indéfiniment ; lorsque les désavantages commenceront à s'accumuler, d'autres objectifs de recherche deviendront dominants, et la taxonomie évoluera dans une direction différente.

Ian Hacking (1995, 1999), qui définit le nominalisme en opposition avec le structurisme inhérent, est un exemple clair d'un philosophe suivant les tendances nominalistes contemporaines. Le structurisme inhérent est la thèse selon laquelle le monde est déjà organisé et arrangé de la bonne façon : il vient à notre rencontre préemballé, et notre travail est de découvrir son organisation. Le structurisme inhérent est ce que le nominalisme n'est pas. Cela ressemble à ce que Lakoff et Johnson (1980) ont nommé le mythe de l'objectivisme en linguistique, mais ils évitent habilement les problèmes associés avec les critiques philosophiques de ce terme couramment utilisé.

Hacking soutient que les catégories que nous adoptons sont basées sur ce que l'on découvre lors des expérimentations - mais il ne croit pas qu'il y ait une seule liste véritable des catégories qui existent dans le monde. Le livre de la nature définitif qui établirait la véritable taxonomie de toutes les catégories naturelles est irréalisable, et cela n'aurait aucun sens, même comme possibilité logique. Hacking n'accorde pas plus d'importance à la philosophie de la représentation qu'à l'intervention lorsqu'il s'agit de décider ce qu'est le monde. Putnam appelle cela " le rejet du point de vue divin ", et Dewey « le rejet du point de vue du spectateur sur la connaissance ».

Le fait qu'il ne soit pas nécessairement anti-réaliste est une autre caractéristique du nominalisme contemporain qui mérite d'être mentionnée. Le nominalisme est anti-réaliste pour ce qui est des essences, non pour ce qui est des espèces. Car il peut exister des espèces réelles sans essence. L'or, les tigres, la peur et les troubles bipolaires sont tous des espèces réelles. Les inférences au sujet d'un membre du groupe peuvent nous renseigner sur les autres membres du groupe, et elles sont importantes au point de vue pratique — c'està-dire qu'elles nous indiquent comment les objets d'une espèce sont censés se comporter lorsque nous interagissons avec eux de façon spécifique. Les essences ne sont pas requises. Techniquement, cependant, il est préférable de dire que ce sont des espèces très pratiques plutôt que de parler d'espèces réelles.

Le " réel " est un mot qui possède plusieurs sens. C'est un outil philosophique ; nous l'utilisons pour évaluer, organiser, privilégier et unifier notre expérience. Quelquefois, réel signifie non imaginaire, comme dans " une licorne est imaginaire et l'holocauste ne l'est pas - il a réellement eu lieu ». Cet usage a souvent des dimensions morales et peut être lié à des arguments philosophiques outranciers. Quelquefois, réel signifie être un élément légitime ou authentique d'une catégorie, comme dans "les baleines sont réellement des mammifères mais le Cheez whiz n'est pas réellement du 
fromage ". Quelquefois, réel est utilisé pour ajouter de l'emphase à une phrase comme dans " Je suis réellement sérieux » ou " Je suis réellement en colère. »

Szasz s'est retrouvé en mauvaise posture pour avoir dit que la schizophrénie n'est pas une véritable maladie mentale. Pour de nombreuses personnes, cette affirmation équivaut à celle qui consiste à nier que l'holocauste a eu lieu. C'est ici que les distinctions philosophiques peuvent nous venir en aide. Szasz ne soutient pas que la schizophrénie est imaginaire, ou qu'elle n'existe pas ; il argumente surtout qu'elle n'est pas une condition médicale authentique. Du point de vue du modèle des espèces pratiques, Szasz a adopté une définition trop conservatrice des conditions médicales, et il existe de bonnes raisons pour classer la schizophrénie dans le cadre du modèle médical.

John Dupré $(1981,1999)$ a créé une controverse en affirmant que les baleines sont des poissons. Pour être charitable envers Dupré, à moins d'être une personne " réellement » rigide, on devrait être capable d'admettre que les baleines sont similaires aux poissons. Lorsqu'on a compris que les mammifères ont le sang chaud et donnent vie à des petits qui sont vivants, il est clair que les baleines sont des mammifères. Il existe de bonnes raisons de classer les baleines ainsi, et Dupré n'a jamais été en désaccord avec ces raisons. Il voulait faire valoir que tout ce qui ressemble à un poisson peut potentiellement être catégorisé comme un poisson dans un quelconque système, lequel peut être utile et légitime pour certaines visées. Cette affirmation gagne en force lorsqu'on remarque que "poisson » n'est même pas un terme proprement scientifique puisqu'il recouvre toutes sortes d'organismes différents. Affirmer que les mammifères ne peuvent être des poissons est une question de stipulation. La même chose est probablement vraie du Cheez whiz et du fromage.

Le modèle des espèces pratiques est une version du nominalisme qui évite ce genre de controverses en évaluant les catégories en fonction de leur valeur marchande ou de leur utilité, non pour leur supposée capacité à représenter toute réalité théorique antécédente. "Utilité » sous-entend la capacité d'agir avec succès. Le modèle permet également que le terme "réel " soit philosophiquement utile et n'adopte pas le dogme anti-métaphysique de l'empirisme conventionnel, mais sa position quant à ce qui compte comme réel est plutôt variée, large, pluraliste et interactive. Bien que le modèle des espèces pratiques ait été présenté comme l'opposé du structurisme inhérent, on peut aussi dire qu'il embrasse le réalisme dévergondé [promiscuous] de Dupré.

\section{Les espèces pratiques et l'éliminativisme}

De la façon dont je l'utilise, à tout le moins, le modèle des espèces pratiques a été développé dans le contexte de l'opposition à l'éliminativiste, même s'il était également marqué par les arguments éliminativistes. Le modèle des espèces pratiques est même ouvert à l'éliminativisme en ce qui concerne les essences et les espèces naturelles.

Les éliminativistes excellent à donner des arguments non essentialistes pour les phénomènes comme la douleur, la conscience, la mémoire, les 
croyances et l'identité individuelle. Souvent, ces arguments entraînent le fractionnement de ce qui paraissait être une catégorie cohérente en sous-catégories, sous le prétexte que ces catégories sont des regroupements vagues de phénomènes différents - qu'elles ne sont pas des espèces scientifiquement légitimes. Après avoir formulé un assez bon argument faisant appel à la complexité, les éliminativistes soutiennent que, si une catégorie n'est pas cohérente ou intégrée, c'est une catégorie arbitraire ou artificielle, donc, pour cette raison, elle doit être éliminée de tout discours scientifique respectable. Souvent, une catégorie n'est pas pertinente du point de vue d'un programme de recherche déjà adopté, par exemple les neurosciences computationnelles.

Le programme d'analyse éliminativiste a malheureusement acquis le statut d'un shibbolèt, la marque de la crédibilité scientifique pour les philosophes. Certains philosophes traitent de l'élimination comme un but a priori et ils adoptent volontairement un jargon éliminativiste désignant péjorativement ce qu'ils veulent éliminer comme étant commun, folklorique, dépassé, arbitraire, artificiel, et tout simplement mauvais. Leurs arguments philosophiques prennent souvent une tournure émotionnelle et un ton dédaigneux.

Dans le modèle des espèces pratiques, la distinction entre la connaissance scientifique et le sens commun n'est pas envisagée comme une chose immuable, mais plutôt fluide (Zachar, 2000c). C'est actuellement un problème de sens commun que de comprendre que la terre est ronde, qu'elle tourne autour du soleil et que nous pensons avec nos cerveaux - mais cela n'a pas toujours été le cas. La croyance que certaines maladies sont causées par des microbes, un autre credo du sens commun, a été ridiculisée par la communauté scientifique lorsque Pasteur l'a proposée. Qualifier quelque chose de commun ou de folklorique n'est pas nécessairement une insulte ou même un argument. En outre, les catégories du sens commun n'ont pas à se conformer aux standards de la classification scientifique pour être légitimes, pensons par exemple aux catégories comme les mauvaises herbes, les familles, les mères et les arbres. Cela vaut également pour les catégories philosophiques comme le nominalisme, le réalisme, l'éliminativisme et les espèces naturelles.

Les mots « arbitraire » et « artificiel » tendent à suggérer que quelque chose est fabriqué ou même aléatoire, mais ils signifient habituellement que l'auteur est en désaccord avec les raisons de la distinction, ou que la distinction n'est pas aussi tranchée qu'il le voudrait. L'affirmation selon laquelle les retards mentaux et l'hypertension sont arbitrairement définis est un bon exemple de cette tendance. Les habiletés intellectuelles générales peuvent être mesurées sur un continuum de faible à élevé, et la différence entre une personne qui a un QI de 70 et une autre qui en a un de 71 est mince - mais choisir un QI de 70 comme seuil du retard mental n'est pas arbitraire. Deux déviations standard en dessous de la moyenne d'un test de QI de Wechsler est un seuil raisonnable. En plus, une limite floue ne signifie pas que le retard mental n'existe pas.

Quand la confrontation avec la complexité amène les penseurs à offrir des interprétations non essentialistes, les deux options populaires sont d'opter 
soit pour l'éliminativisme, soit pour le nominalisme. Les partisans du modèle des espèces pratiques préfèrent l'option nominaliste. Par certains aspects importants, ils sont proches des éliminativistes, car ils acceptent plusieurs de leurs arguments tout en refusant leurs conclusions austères.

\section{Préoccupations et complication}

Une préoccupation courante concernant le modèle des espèces pratiques est qu'il ratisse trop large, c'est-à-dire que tout en vient à être considéré comme une espèce pratique. La critique est importante et, d'une certaine façon, juste. Le modèle des espèces pratiques est une théorie générale de la classification. Il ne dresse pas de liste des différentes espèces comme les espèces artificielles, les espèces appropriées, les espèces fonctionnelles, les espèces interactives, les espèces relationnelles, les espèces taxinomiques, les espèces naturelles, puis, y ajoute les espèces pratiques. Il soutient plutôt que la pragmatique de la classification ne peut être éliminée de l'évaluation des espèces. En d'autres mots, les considérations pragmatiques sont pertinentes en tout temps et pour toutes les espèces. Au regard de ces considérations pragmatiques, il existe un continuum conceptuel, concernant le caractère pratique, qui va du bas vers le haut. "Pratique " dans ce contexte signifie tout ce qui nous permet de réussir compte tenu des différentes positions que nous avons prises. Sans conteste, " pratique » est un terme vague, et il recouvre beaucoup de types différents d'évaluation. Bien que l'endroit où quelque chose sera placé dans le continuum soit relatif au contexte plutôt que fixé à l'avance, le continuum des espèces pratiques est à peu près similaire à la caractéristique des « espèces nominales » par rapport aux " espèces naturelles » de Keil (1989).

L'endroit où se situe, dans le continuum, un concept particulier d'espèce dépend du type de problème que l'utilisateur tente de résoudre, c'est-à-dire que la validité pratique d'une espèce est définie de la même façon que les psychométriciens définissent la validité. Celle-ci est une évaluation de la légitimité des inférences obtenues à partir des résultats des tests, et chaque inférence doit être soutenue par des preuves. Il n'y a pas de chose telle que la validité d'un test, il existe plutôt une validité pour différents usages du test.

Dans le cas où les espèces sont des groupes d'individus au sujet desquels nous faisons des inférences, les mêmes principes s'appliquent pour évaluer la validité de celles-ci. Donc, plus une espèce peut être utilisée dans la résolution de divers types de problèmes et dans différents programmes de recherche, plus elle est pratique. La qualité pratique ne devrait pas être confondue avec l'intérêt utilitariste à court terme qui s'occupe uniquement des expédients. Comme l'a dit James, le plus important, c'est la validité à long terme, et elle est souvent différente de ce qui constitue un expédient à l'heure actuelle.

Cela ne veut pas dire qu'il n'existe qu'un seul type d'espèce dans le monde - l'espèce pratique. Il est utile de distinguer différents types d'espèce. Tout groupe qui comporte quelque règle d'inclusion peut être considéré comme une espèce. Des groupes comme les choses blanches, les lions ou les capricornes 
ne sont pas des espèces très utiles. On pourrait les nommer des non-espèces, mais cela signifierait simplement qu'ils ne sont pas très utiles (ou seulement un peu) dans la pratique. Comme le note Haslam (2002), certaines espèces, telles que le retard mental ou la dépression, sont en quelque sorte découpées dans le continuum. Les points de découpe entre les cas qui font partie de l'espèce et ceux qui n'en font pas partie ne peuvent être choisis en utilisant les propriétés inhérentes à la catégorie. Dans d'autres espèces, il y a des limites distinctes entre les membres et les non-membres, même si ces limites sont floues ou s'il existe des cas intermédiaires. Les recherches de Haslam indiquent que les troubles de personnalité des « cas-limite " exemplifient ce patron. Dans certaines espèces également on observe des limites discrètes entre membres et non-membres sans cas intermédiaires - comme dans la dépression mélancolique et le syndrome de Williams. Il existe donc de nombreuses espèces d'espèce.

À l'origine, le modèle des espèces pratiques a été présenté comme un rejet des espèces naturelles. Le problème quant à cette façon de justifier le modèle est que les " espèces naturelles » sont difficiles à définir (tout comme les "véritables essences »). Évidemment, certaines espèces, comme les tigres, existent naturellement, mais ce n'est pas le cas pour d'autres, comme les ordinateurs. Voilà une distinction potentiellement utile, mais certaines catégories de choses qui existent naturellement, tels les poissons et les arbres, ne sont pas à proprement parler des espèces naturelles, tandis que certaines choses non naturelles, tels le radium et les antibiotiques, ressemblent plus à des espèces naturelles qu'à des artefacts. Des philosophes comme Griffiths (1997) optent pour une approche externaliste afin de définir les espèces naturelles et affirment que les éléments chimiques et les espèces animales sont des espèces naturelles ; donc, peu importe ce que l'on veut dire par " être une espèce naturelle ", c'est ce qu'ils sont. C'est une proposition intéressante en soi, mais qui n'est pas non plus ce à quoi s'oppose le modèle des espèces pratiques. La notion de "groupe de propriétés homéostatiques déterminées causalement ", de Boyd (1991), a, elle aussi, du mérite, mais elle peuple également le monde avec d'innombrables hiérarchies d'espèces naturelles.

Les espèces naturelles au sens de Putnam-Kripke sont une version du structurisme inhérent selon lequel une espèce existe dans le monde de façon telle que sa microstructure essentielle est perceptible par les scientifiques. Tout terme qui réfère à cette espèce est un indicateur rigide — ce qui signifie qu'il réfère à cette espèce dans tous les mondes possibles, même si l'utilisateur du langage n'a pas connaissance de la microstructure essentielle. Ce que fait la science, c'est de nous enseigner la véritable signification ou le référent de nos termes d'espèce. Dans ce modèle, les espèces sont là dans le monde, et nous interagissons avec elles. Une fois que nous avons identifié et nommé ses membres, nous avons nommé l'espèce, même si nous ne comprenons pas complètement ce qu'elle est. Voilà ce à quoi le modèle des espèces pratiques s'oppose. 
Bien qu'elles portent principalement sur l'individuation des contenus mentaux, les expériences de pensée comme celles de la Terre jumelle peuvent être envisagées comme portant également sur la nature des espèces. Un argument habituel, quant à la classification, contre les expériences de la Terre jumelle est que les analyses fonctionnelles sont aussi pertinentes pour décider de ce qui compte comme espèce particulière. Cet argument est compatible avec le modèle des espèces pratiques. $\mathrm{Si}_{2} \mathrm{O}$ et $\mathrm{XYZ}$ ne peuvent être fonctionnellement distingués, alors ils sont tous deux de l'eau. Sans autres différences pratiques entre $\mathrm{H}_{2} \mathrm{O}$ et XYZ, il n'y a pas de bonnes raisons d'en faire différentes espèces de choses. Ils sont de la même espèce. Conformément à la définition essentialiste des troubles de Wakefield, si un organisme est adaptatif dans un type d'environnement et développe une anomalie constitutive mais qu'il est ensuite placé dans un autre environnement dans lequel son anomalie est adaptative, alors il n'y a pas trouble, et, à la génération suivante, pas d'anomalie. C'est ainsi qu'opère la sélection naturelle. En outre, si un organisme fonctionne selon certains paramètres constitutifs mais qu'il sont une variante d'une mésadaptation particulière à un environnement particulier et que d'autres organismes sont des variantes plus adaptées, alors le comportement mésadapté peut être considéré comme un trouble si un traitement peut améliorer son fonctionnement.

Le modèle des espèces pratiques ne nie pas qu'un élément que nous appelons « or » et dont le noyau comprend 79 protons était, il y a cinq cents ans, identique à qu'il est aujourd'hui, même si les gens ne pouvaient pas toujours identifier très exactement cette matière. Il ne s'objecte pas non plus au fait de se fonder sur des considérations externalistes pour décider à quoi réfèrent les termes. Ce qu'il nie, c'est que le mot " or " doive, selon les usages antérieurs, référer à cet élément dans tous les mondes possibles. Ce pourrait bien ne pas être le cas. Le monde est un endroit complexe, et l'élément dont le noyau comporte 79 protons pourrait, à certaines fins, être conçu comme différentes espèces de choses ou en tant que partie d'une autre espèce de choses, de la même manière que l'élément dont le noyau comprend un proton est actuellement vu comme plusieurs espèces différentes de choses.

Si une telle pragmatique de la classification s'applique à quelque chose d'aussi stable et « indifférent » que les éléments chimiques, elle s'applique d'autant plus fortement à une chose aussi dynamique, fluide et influencée par les rapports interpersonnels que les troubles psychiatriques. On peut le constater avec la schizophrénie. Selon Paul Meehl (1962, 1972, 1995), la schizotaxie est une catégorie à laquelle les personnes peuvent ou non appartenir. Dans sa forme la moins sévère, elle peut être décrite comme une condition connue sous le nom de schizotypie, alors que dans les cas de plus grande mésadaptation elle est appelée schizophrénie. Les diverses manifestations pathologiques de la schizotaxie sont collectivement connues comme le spectre schizophrénique. Posséder un certain ensemble de gènes particuliers ne signifie pas que quelqu'un a un trouble psychiatrique, mais qu'il est plus susceptible 
d'en développer. Quelques personnes dans ce groupe peuvent être étranges ou excentriques mais ne souffrir d'aucun trouble.

Décider où commence la schizophrénie dans la catégorie de la schizotaxie requiert un jugement évaluatif. Nous pouvons décider de définir la schizophrénie de façon large ou étroite. Si nous la définissons de façon large, nous identifierons comme "schizophrénie " un ensemble diversifié de groupes de comportements. Si nous la définissons de façon étroite, certaines personnes éprouvant des hallucinations et des illusions pourraient ne pas être classées comme schizophrènes. Une fois que nous avons décidé des limites de la catégorie, quelqu'un peut dire que la schizophrénie est une véritable espèce, s'il insiste pour s'exprimer ainsi ; cependant, distinguer la catégorie de la schizophrénie des autres catégories dans le spectre n'est pas seulement une affaire de découverte, cela requiert des décisions. La schizophrénie est une espèce pratique.

(Traduction de Davy Mougenot)

\section{Bibliographie}

Andreasen, N.C. The Broken Brain : the Biological Revolution in Psychiatry, New York, Harper \& Row Publishers, 1984.

Boyd, R. « Realism, Anti-Foundationalism, and the Enthusiasm for Natural Kinds ", Philosophical Studies, 61, 1991, p. 127-148.

Bryant, R. Discovery and Decision, Londres, Associated University Presses, 2000.

Canfield, J.V. « Discovering Essence ", dans C. Ginet et S. Shoemaker, dir., Knowledge and Mind, New York, Oxford University Press, 1983, p. 105-129.

Donnellan, K.S. "Kripke and Putnam on Natural Kind Terms », dans C. Ginet et S. Shoemaker, dir., Knowledge and Mind, New York, Oxford University Press, 1983.

Dupré, J. « Natural Kinds and Biological Taxa », Philosophical Review, 90, 1981, p. 6690.

- The Disorder of Things, Cambridge, Mass., Harvard University Press, 1993.

Gelman, S.A. et J.D. Coley. « The Importance of Knowing a Dodo is a Bird : Categories and Inferences in 2-Years Old Children ", Developmental Psychology, 26, 1990, p. 796-804.

Gelman, S.A. et H.M. Wellman. « Insides and Essences : Early Understandings of the Non-Obvious ", Cognition, 38, 1991, p. 213-244.

Gelman, S.A. et L.A. Hirschfeld. « How Biological is Essentialism », dans D.S. Medin et S. Atran, dir., Folkbiology, Cambridge, Mass., MIT Press, 1999, p. 403-446.

Ghaemi, S.N. The Concepts of Psychiatry, Baltimore, The Johns Hopkins University Press, sous presse.

Griffiths, P.E. What Emotions Really Are, Chicago, University of Chicago Press, 1997.

Goodman, N. Ways of Worldmaking, Indianapolis, Hackett Publishing Company, 1978.

Grove, W.M. et A. Tellegen. "Problems in the Classification of Personality Disorders ", Journal of Personality Disorders, 5, 1991, p. 31-41.

Guze, S. Why Psychiatry is a Branch of Medicine, New York, Oxford University Press, 1992.

Hacking, I. Rewriting the Soul : Multiple Personality and the Sciences of Memory, Princeton, New Jersey, Princeton University Press, 1995. 
- The Social Construction of What ?, Cambridge, Mass., Harvard University Press, 1999.

Haslam, N. "Kinds of Kinds : a Taxonomy of Psychiatric Categories ", Philosophy, Psychiatry, and Psychology, 9, 2002, p. 203-217.

Keil, F.C. Concepts, Kinds, and Cognitive Development, Cambridge, Mass., The MIT Press, 1989.

Laing, R.D. The Divided Self, Londres, Tavistock Publications, 1960.

Lakoff, G. Women, Fire, and Dangerous Things, Chicago, the University of Chicago Press, 1987.

Lakoff, G. et M. Johnson. Metaphors we Live By, Chicago, the University of Chicago Press, 1980.

Meehl, P.E. "Schizotaxia, Schizotypy, Schizophrenia ", American Psychologist, 17, 1962, p. 827-838.

—. "Specific Etiology, Psychodynamics, and Therapeutic Nihilism », International Journal of Mental Health, 1, 1972, p. 10-27.

- "Bootstraps Taxometrics ", American Psychologist, 50, 1995, p. 266-275.

Mill, J.S. "A System of Logic ", dans J. M. Robson, dir., Collected Works of John Stuart Mill Vol. VII, Toronto, University of Toronto Press, 1843, 1973.

Ortony, A. et T.J. Turner. "What's Basic About Basic Emotions ? ", Psychological Review, 97, 1990, p. 315-331.

Szasz, T. The Myth of Mental Illness, New York, Harper Row, 1961.

Wakefield, J.C. "The Concept of Mental Disorder : on the Boundary Between Biological Facts and Social Values ", American Psychologist, 47, 1992, p. 373388.

—. "Aristotle as Sociobiologist : the 'Function of a Human Being' Argument, Black Box Essentialism, and the Concept of Mental Disorder ", Philosophy, Psychiatry, and Psychology, 7-1, 2000, p. 17-44.

Wender, P.H. et D.F. Klein. Mind, Mood, and Medicine, New York, Farrar, Strauss, Giroux, 1981.

Widiger, T.A. et A.J. Francis. "Toward a Dimension Model for the Personality Disorders ", dans T. Costa et T.A. Widiger, dir., Personality Disorders and the Five-Factor Model of Personality, Washington, D.C., American Psychological Association, 1994, p. 19-39.

Zachar, P. "Psychiatric Disorders are not Natural Kinds ", Philosophy, Psychiatry, and Psychology, 7, 2000a, p. 167-182.

—. "Folk Taxonomies Should not Have Essences Either », Philosophy, Psychiatry, and Psychology, 7, 2000b, p. 191-194.

- Psychological Concepts and Biological Psychiatry : a Philosophical Analysis, Amsterdam, John Benjamins, 2000c.

- - " The Practical Kinds Model as a Pragmatist Theory of Classification ", Philosophy, Psychiatry, and Psychology, 9, 2002, p. 219-227. 